\title{
Cooperative Group Behavior Research Based on Lattice
}

\author{
Linlin Shen*1, Jingbo Zhou ${ }^{1}$, Longcheng Xing ${ }^{1}$, Xiaodong Pan ${ }^{1}$, Yu Zhang ${ }^{2}$ \\ ${ }^{1}$ College of Civil Engineering and Architecture, Hebei University, Baoding, China \\ ${ }^{2}$ China Architecture Standards Design Institute \\ Email: $769712060 @ q q . c o m$
}

\begin{abstract}
This paper mainly studies the cooperative evolution of game model on the regular lattice, and to explore factors that influence the evolution of cooperation, including proportion of initial betrayers, the game model, the profit ratio, the number of neighbors that profit information, and the policy update rules. The results show that: Different factors have different effects on the evolution of cooperation. The proportion of initial traitors has no effect on the evolution of cooperation (except in extreme cases). The profit ratio has a great influence on the evolution of cooperation. The different profit ratio has a direct impact on the stability of cooperation evolution, and the stable state of the profit ratio between 1.8 and 2.0 is almost the same. Policy update rules are the most important factors affecting the evolution of cooperation, and different rules determine the ratio of cooperation when the evolution of cooperation is stable. With the increase in the number of neighbors that profit information, the ratio of cooperation is steadily rising when cooperation is stable. Prisoner's dilemma is more cooperative than snowdrift game.
\end{abstract}

Keywords: Rules lattice; influence factor; game; evolution of cooperation

\section{Introduction}

Cluster movement is a common natural phenomenon, such as swarm flying of birds, swarm parade of fish, collective migration of locusts, as well as the emergence of the crowd on various occasions are typical representative of the cluster movement [1]. In fact, all kinds of coordinated and orderly collective movement patterns presented by biological groups are emergent behaviors resulting from simple local interactions among individuals. Cooperation plays a vital role in both the evolution of biological populations and the development of human society. But selfish altruistic behavior between individuals contradicts Darwin's theory of natural selection. How to cooperate between selfish individuals is a problem that people have always been concerned about and studied. Evolutionary game theory provides a good platform for this problem, and evolutionary game involves mathematics, physics, control theory, economy and other research fields. The network topology provides an interactive way for evolutionary game theory, which makes the evolution of cooperation more realistic.

Since the 1990s, evolutionary game theory has developed rapidly. Before that, the idea of evolution has begun to germinate in the middle of the last century that mainly by static analysis. In 1948, Marshall pointed out that evolution is more complex than static analysis [3]. In 1950, Nash argued that the Nash equilibrium could be achieved by assuming that the participants could obtain all the relative information when the various pure strategies were adopted. This group behavior interpretation was regarded as the earliest evolutionary game theory. Smith and Price put forward the evolutionary stability strategy in evolutionary game theory $[4,5]$. They drew people's attention from the theory of game theory, so that people began to provide new ideas for the study of evolutionary game theory from the perspective of bounded rationality. In the 1980s, with the deepening of evolutionary game theory, many economists introduced evolutionary game theory into the economic field to analyze the stock market, industrial evolution and so on.

In 90s, evolutionary game theory entered a new stage of research. The Weibull system summarized the evolutionary game theory [6]. Bester and Guth use the knowledge of evolutionary game theory to study the existence of altruistic behavior and its evolutionary stability in economic activities. In 2000 Gutlman used game evolution theory to study whether reciprocity can exist in opportunism [8]. Daniel, Arce and Todd studied four different prisoner dilemma models, and obtained the evolution and information requirements of individual cooperation of the four prisoner dilemma models [9]. And the evolutionary game is used to 
analyze the specific problems such as the environmental regulation strategy of local governments[10], the behavior of group bidding experts[11], and the diffusion of green technological innovation of enterprises[12]. However, the research on evolutionary game theory is not comprehensive enough. Paper studies the evolution of game model on a regular lattice, and to explore factors that influence the evolution of cooperation, including proportion of initial traitors, the game model, profit ratio, the number of neighbors' profit information, and update rules.

\section{Evolutionary Game Theory}

Game refers to the process that under certain conditions and rules, people or organizations decide their own behavior by choosing a certain strategy, so as to obtain their own benefits. A game usually consists of 4 parts: game individual, strategy set, game profit, strategy evolution, and [13]. Game individual refers to a person or an organization that changes strategy and gains profit in the process of gambling. Strategy set is a strategy adopted by individuals in game playing. Game profit refers to the gains and losses of game players after game. Strategic evolution refers to the way in which the game adjusts its strategy according to the principle of maximizing its own profit. In the 1950s, John Nash, an American scientist, proposed an important concept in classical game theory, Nash Equilibrium [14]. The core idea is that for two or more players, the evolution of individual strategy will tend to an equilibrium state, and no individual can get higher profits by changing his own strategy. But the individual Nash equilibrium does not represent the Nash equilibrium of the whole population. The following two classical game models are used to understand Nash equilibrium in depth.

Prisoner's dilemma game [15]: If two thieves were a gang, they were caught by the police at the same time. In separate trials, if neither person identifies the other's crime, both parties are sentenced to one year's imprisonment. The police therefore said that if the offender was identified and the offender did not identify himself, he would be acquitted and the other would be sentenced to three years' imprisonment, but if both parties identified the offender, they would be sentenced to two years' imprisonment. Both sides are faced with the temptation of acquittal, so the two sides are caught in the game. Cooperation between the two sides will yield R. When one side of the cooperation betrayed, the Betrayer gains T, and the cooperator gains $\mathrm{S}$. Betray between the two sides will yield $\mathrm{P}$. The profit relationship is $\mathrm{T}>\mathrm{R}>\mathrm{P}>\mathrm{S}$. The profit matrix is shown in Table 1. According to this profit matrix, we set $\mathrm{R}=1, \mathrm{~T}=\mathrm{b}, \mathrm{S}=\mathrm{P}=0$ [16]. Because $\mathrm{R}=1$, $\mathrm{b}$ is the profit ratio. Since the profit of the cooperator relative to the Betrayer is less than that of the betrayer, the betrayer is the best decision no matter what strategy the other party chooses. Then the choice of betrayer will continue until there is no cooperator in the population. At this time, the individual has no profit, and the overall profit is 0 , which is in equilibrium. This is the origin of the dilemma, and selfish behavior of individuals brings disaster to the population.

Table 1. Prisoner's dilemma income matrix.

\begin{tabular}{ccc}
\hline & cooperation & betray \\
\hline cooperation & $(\mathrm{R}, \mathrm{R})$ & $(\mathrm{T}, \mathrm{S})$ \\
betray & $(\mathrm{T}, \mathrm{S})$ & $(\mathrm{P}, \mathrm{P})$ \\
\hline
\end{tabular}

Table 2. Snowdrift game income matrix.

\begin{tabular}{ccc}
\hline & cooperation & betray \\
\hline cooperation & $(1,1)$ & $(\mathrm{b}, 2-\mathrm{b})$ \\
betray & $(2-\mathrm{b}, \mathrm{b})$ & $(0,0)$ \\
\hline
\end{tabular}

Snow drift game [17]: If two persons drive in opposite directions on a newly snowy road and are blocked by a heavy snowdrift, they face the work of shoveling the snow. If the total amount of labour to be removed from the snow drift is $\mathrm{c}$, the convenience to get home smoothly is $\mathrm{b}(\mathrm{b}>\mathrm{c})$. When two people remove snow drifts together, everyone gains $\mathrm{R}=\mathrm{b}-\mathrm{c} / 2$. When a person gets off and shovels and waits for a person to wait in the car, the shoveler's profit is $\mathrm{S}=\mathrm{b}-\mathrm{c}$, and the waiting person's profit is $\mathrm{T}=\mathrm{b}$. Both sides do not get off to shovel snow, so the profit are $\mathrm{P}=0$. The profit relationship is $\mathrm{T}>\mathrm{R}>\mathrm{S}>\mathrm{P}$. Assuming $\mathrm{b}-\mathrm{c} / 2=1$, then $\mathrm{c}=$ 
$2 \mathrm{~b}-2, \mathrm{~b}-\mathrm{c}=2-\mathrm{b}$, the payment matrix is shown in Table 2 , where the value of $\mathrm{b}$ is the profit ratio and $1<$ $\mathrm{B}<2$. Because the profit of the cooperator is greater than that of the betrayer, it is more conducive to cooperation. The cooperator will exist and the population will not die out.

Classical game theory assumes that individuals are completely rational and fully understand the profit matrix. However, in this case, all individuals will adopt Nash equilibrium, resulting in irrational groups. In real life, individuals in complex environments do not have the ability to choose strategies that maximize their own interests. That is to say, individuals only control local information to take the best strategy to maximize their own interests, and individuals are bounded to be rational in the process. Evolutionary game theory is that individuals maximize their own interests through learning in the process of repeated games under bounded rationality.

\section{Game Analysis on Regular Lattice}

In reality, the contact between individuals is always limited, and the individual contacts only a few other individuals around it. Therefore, in the theoretical analysis of the relationship between individuals in the game, it is generally believed that there is a certain topological network between individuals. In this paper, we choose the rule lattice as the topology network. The influence of initial traitors, the game model, benefit ratio, the number of neighbors that profit information, and update rules on evolutionary game is analyzed. The rule lattice is shown in Figure 1.

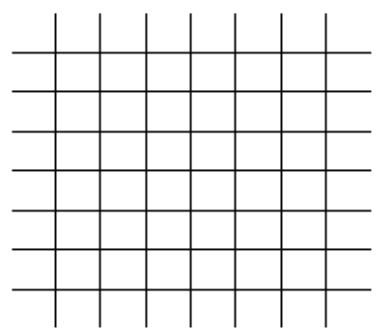

Figure 1. Regular grid graph.

Place the individual game on a two-dimensional rule lattice, that is, a lattice represents an individual. First, the individual game with eight neighbors directly adjacent to the game, and cumulative calculation of each game individual income, and then update the strategy according to certain evolution rules, each game individual adopts the corresponding strategy, however. Then calculate the proportion of coauthors in all individuals.

MATLAB algorithm flow:

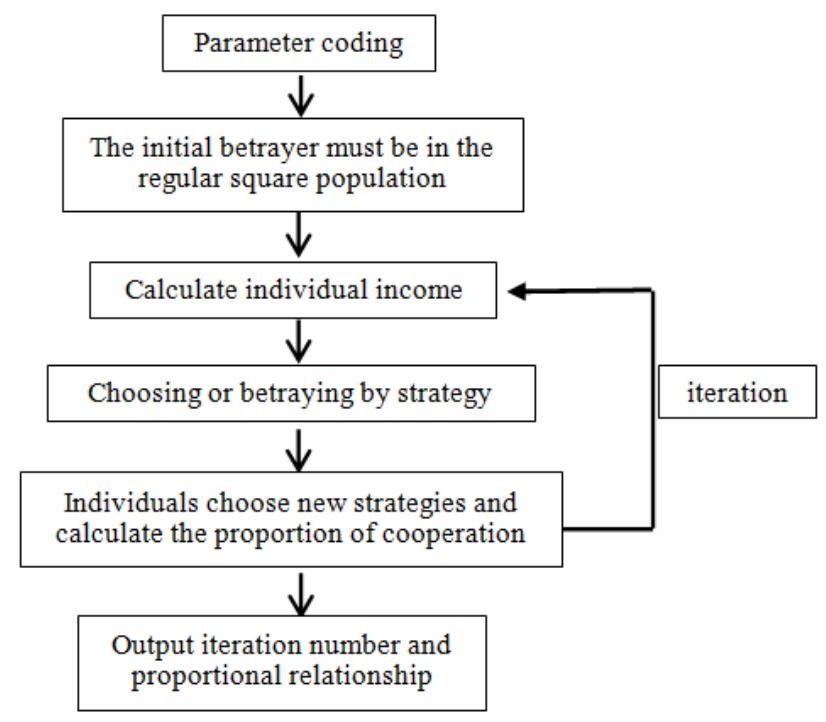




\subsection{Cooperation Evolution of Two Game Models under Different Proportion of Initial Betrayers}

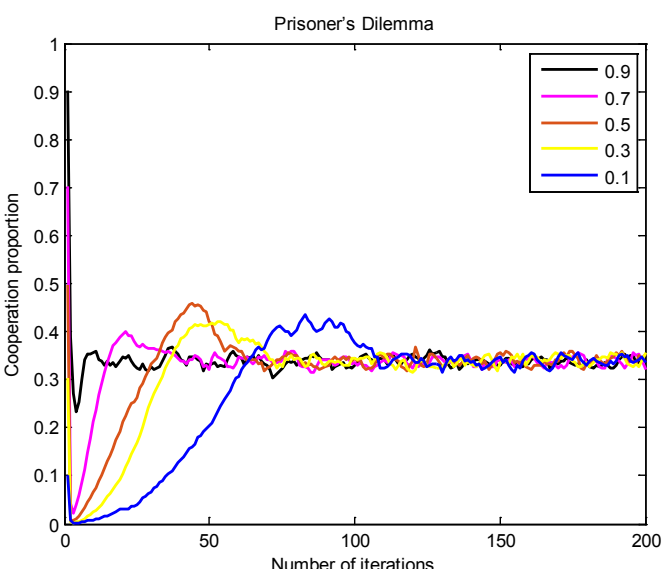

a)

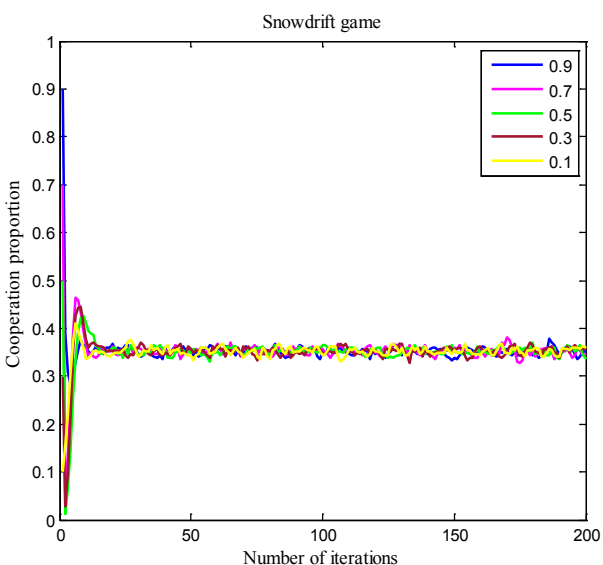

b)

Figure 2. The proportion of different initial betrayer ratio

Fig. 2 shows the relationship between the initial betrayer ratio and the cooperative ratio when the prisoner's dilemma and the snowdrift game remain unchanged in other cases. Among them, the profit ratio is 1.8 , and the information of the eight neighbors is obtained, and the best actor strategy is adopted. The proportionality of the prisoner's dilemma in the stable evolution of the regular lattice is shown in Fig. 2a. After a certain number of iterations, the final proportions of cooperator fluctuate within the same range regardless of the proportion of initial betrayers. So there is no connection between the proportion of cooperation and the ratio of initial betrayers. From Fig. 2 b, we can see that the snow drift game also has the same rule. Therefore, regardless of the initial betrayer ratio (except for extremes 0 and 1 ), there is no effect on the cooperative ratio of evolutionary games.

\subsection{Cooperation Evolution of Two Game Models under Different Profit Ratios}
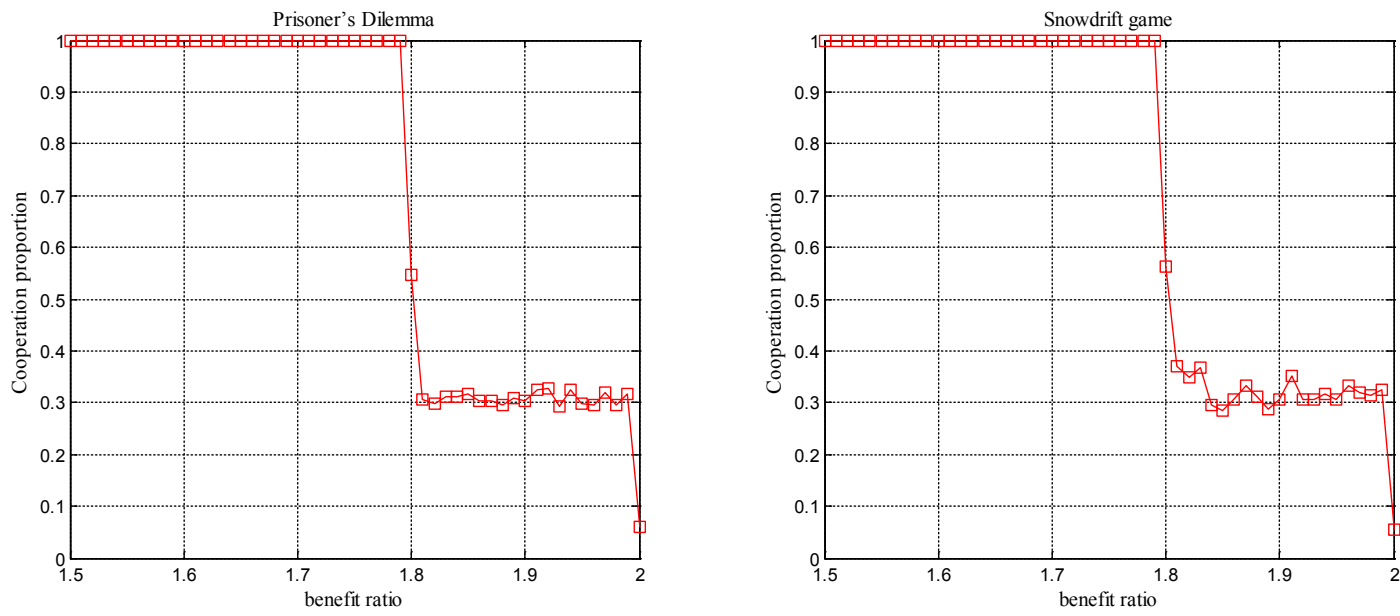

Figure 3. The ratio of cooperation under different profit ratios.

Fig. 3 shows the relationship between the profit ratio of prisoner's dilemma and snowdrift game and the cooperative ratio when other conditions remain unchanged, in which the initial betrayer ratio is 0.1 , the profit information of eight neighbors is obtained, and the strategy of imitating the optimal one is adopted. It is clear that when the profit ratio of the prisoner's dilemma is less than 1.8, the individuals on the lattice 
tend to cooperate basically and there are few betrayers. When the ratio is 2 , the individuals on the lattice tend to be betrayed, and the cooperators are few. In other words, when the profit ratio is very small, the individual still tends to cooperate. With the increase of temptation, the individual gradually changes his strategy. When the temptation becomes very large, the individual almost choose to betray. But when the profit ratio is between 1.8 and 2, the ratio of the cooperators and the betrayers will coexist within a certain range. And the individuals of snow drift game also have the same rule. It can be seen that the profit ratio plays an important role in the stability of evolutionary game, which directly determines whether the cooperation of evolutionary game can be carried out smoothly.

\subsection{Cooperation Evolution of Two Game Models under Different Update Rules}

Update rules are the strategies that individuals adopt to maximize their own interests in the game process. This paper analyzes the influence of the two game models on the evolution of cooperation under the strategy of imitating the best player and the strategy of imitating the winner. Imitate the best player strategy: the individual knows all the information about his neighbor's profit, and then compares it, choosing the strategy of the individual with the highest profit to use in the next round of the game. Imitate the winner strategy: The individual knows all the information about his neighbor's profit, then compares it, remembers the strategies of those individuals whose profit is higher than him, and then uses one of the strategies in the next round of the game with a probability proportional to their profit.
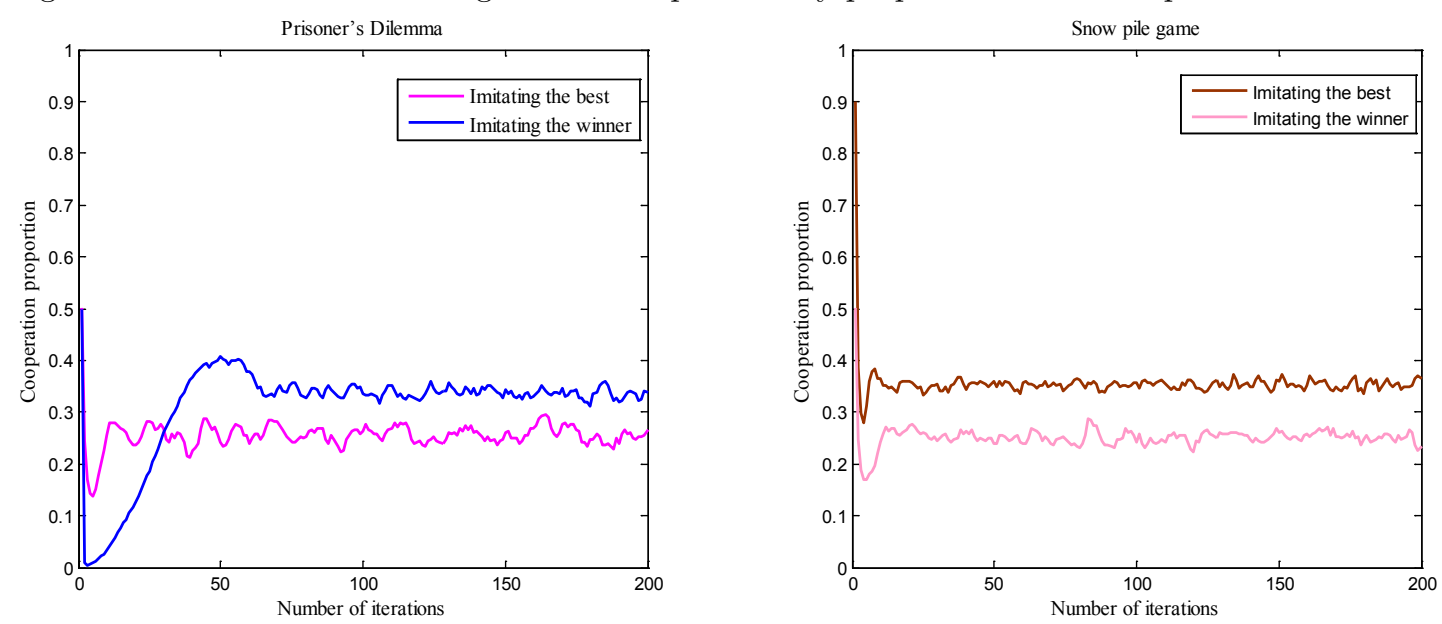

Figure 4. Cooperation ratio under two strategies.

Fig. 4 shows the relationship between the update strategy and the cooperative ratio when the prisoner's dilemma and snowdrift game remain unchanged in other cases. The profit ratio is 1.9 and the initial betrayer ratio is 0.1 . The profit information of eight neighbors is obtained. It can be clearly seen that the evolutionary game of prisoner's dilemma in the rule lattice adopts two strategies under the cooperation ratio, and imitating the best player is the strategy which is beneficial to cooperation. The imitation winner is not conducive to the production of cooperators compared with the imitation the best player. When the evolution is stable, the imitation winner is lower than the imitation of the best player's proportion of cooperation. The snowdrift game has the same rule, and the strategy update rule plays an important role in the evolution of two-dimensional regular lattice.

\subsection{Explore the Cooperation Evolution of Prisoner's Dilemma Game in Different Number of Neighborhood Profit Information}

Fig. 5 is the Prisoner's Dilemma and Snowdrift Game, in other cases unchanged, to understand the relationship between the number of neighbors' profit and the proportion of cooperation when the evolution is stable, in which the profit ratio is 1.9 , the initial betrayer ratio is 0.1 , and the imitation the best player is adopted. It can be clearly seen that the evolutionary game of prisoner's dilemma in the regular lattice increases with the number of neighbors the individual knows, and the proportion of cooperation increases 
when the evolution is stable. And snow drift game also has the same rule. That is to say, when the individual fully understands the neighbor's profit, it will promote the cooperation, and understanding the neighbor's profit will have a greater impact on the evolution of cooperation.
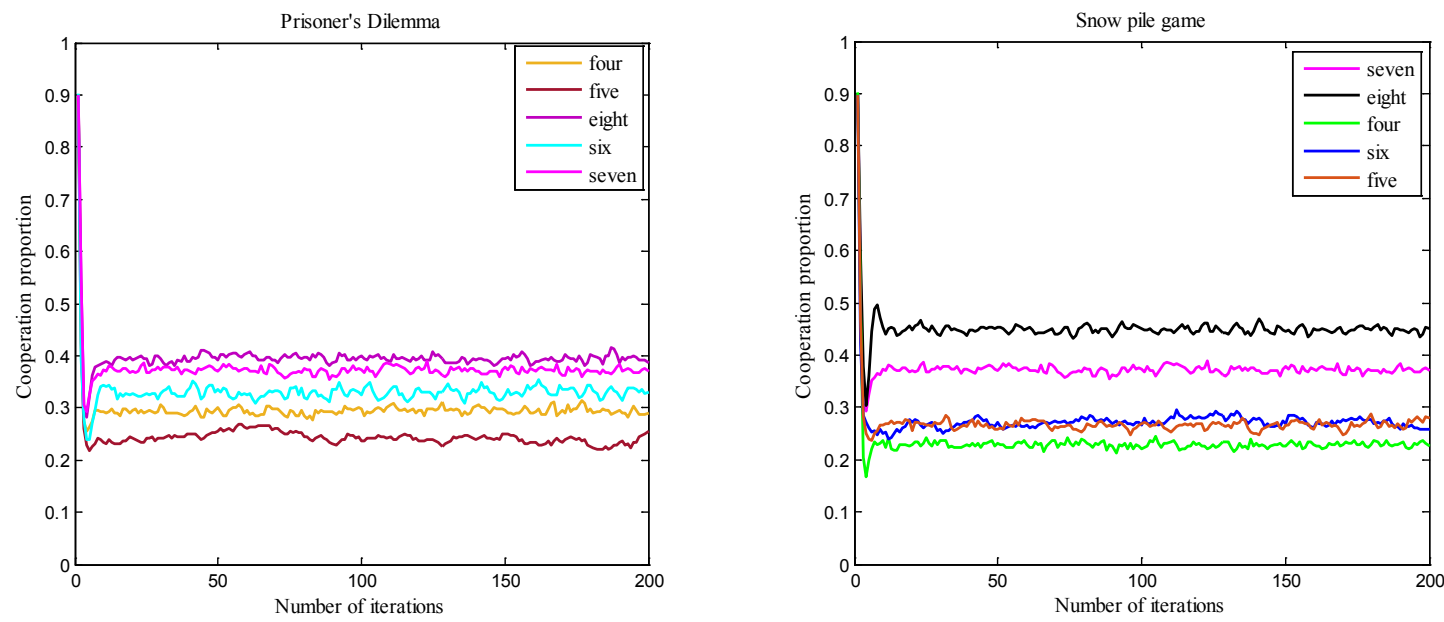

Figure 5. Understanding the cooperation ratio of different neighbors' profit.

\subsection{Cooperation Contrasts between Prisoner's Dilemma and Snow Drift Game}

Through the above analysis, we find that the Prisoner's Dilemma Game adopts the best imitator in the rule lattice, the proportion of the initial betrayer is random, the profit ratio is 1.9, the neighbor's profit information is fully understood, and the cooperation ratio reaches the highest level. The snowdrift game adopts the best imitator in the rules lattice, the initial betrayer proportion is random, the profit ratio is 1.9, the neighbor's profit information is fully understood, the cooperation ratio reaches the highest level. The following is a comparative analysis of the highest level of two game models achieving stable evolution.

In Figure 6 above, we find that when the evolution of the prisoner's dilemma game is stable, the cooperators will unite to resist the intrusion of the betrayers. In the snow drift game, when the regular grid evolves to be stable, the cooperators are more dispersed. It can be seen that the prisoner's dilemma game itself is not conducive to cooperation, but the regular lattice structure has a significant role in promoting his cooperation. For snowdrift game, which is beneficial to cooperation, the rules lattice does not have the promotion to it and the cooperation.

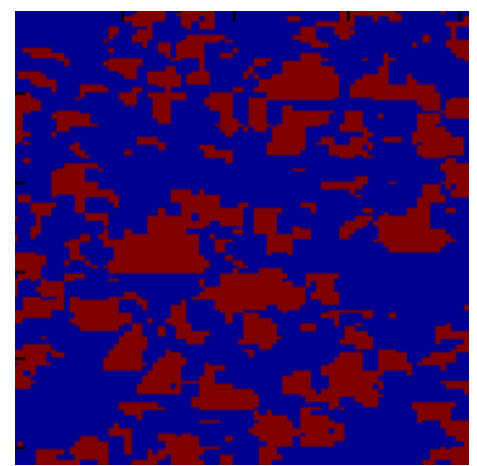

Prisoner's dilemma

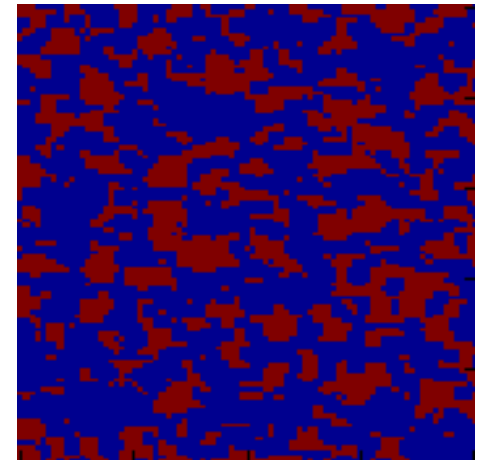

Snowdrift game

Figure 6. Distribution of stable authors after evolutionary games.

\section{Conclusion}

Through the analysis, we know that the evolution of cooperation on regular grid is affected by various aspects. The profit ratio, the number of neighbor profit information and the policy update rules play an 
important role. (1) Profit ratio has a great influence on the evolution of cooperation. Different profit ratios have a direct impact on the stability of cooperation evolution. The stable state of profit ratio between 1.8 and 2.0 is almost the same, but when the profit ratio is greater than 2 and less than 1.8 , it cannot reach a stable state. (2) Updating the rules, different strategies are the most important factors affecting the evolution of cooperation, and different strategies determine the proportion of cooperation when the evolution of cooperation is stable. (3) The number of neighbor profit information, as the number of neighbors' profit information increases, the proportion of cooperation increases when cooperation is stable. Evolutionary game on the rule lattice is not conducive to the cooperation of prisoner's dilemma game itself, and this structure has a significant role in promoting his cooperation, while for snowdrift game, which is conducive to cooperation, the rules lattice does not have the promotion to it and the cooperation.

\section{Reference}

1. Liu Mingyong, Lei Xiaokang, Yang Panpan et al. Theoretical Modeling and Empirical Analysis of Cluster Movement [J].Science Bulletin, 2014 (25): 2464-2483

2. Jiang Tengxu. Overview of intelligent optimization algorithms [J]. computer knowledge and technology: academic exchanges, 2007, 2 (8): 221 222+244

3. Marshall A. Principle of Economics[J]. Germany, the UK and the USA", in67.Fall:519 524

4. Smith J M. The Theory of Games and Evolution of Animal Conflicts[C]. David K. Levine , 2010

5. Smith J M, Price G R. The logic of animal conflict[J]. Resonance, 2010, 10(11):5 5

6. Weibull J W. Evolutionary Game Theory[J]. Games \& Economic Behavior, 1995

7. Bester H, Güth W. Is Altruism Evolutionary Stable?[J]. Journal of Economic Behavior \& Organization, 1994, 34(2):193 209

8. Guttman J M. On the evolutionary stability of preferences for reciprocity[J]. European Journal of Political Economy, 2000, 16(1):31 50

9. Daniel G, Arce M, Todd S. The Dilemma of the Prisoners' Dilemmas[J]. Kyklos, 2005, 58(1):3 24

10. Pan Feng, Xibao, Wang Lin.Evolutionary Game Analysis of Environmental Regulation Strategies among Local Governments[J].China Population, Resources and Environment, 2014, 24 (06): 97-102.

11. Liu Xuwang, Wang Dingwei.Evolutionary Game Analysis of Expert Behavior in Group Bid Evaluation[J].Journal of Management Science, 2015,18(01): 50-61.

12. Cao Xia, Zhang Lupeng.Evolutionary Game Analysis of Enterprise Green Technology Innovation Diffusion[J].China Population, Resources and Environment, 2015,25(07): 68-76.

13. Wu Zhixi, Rong Zhihai, Wang Wenxu. Game mechanics on complex networks [J]. mechanics progress, 2008, 38 (6): 794 804

14. Watts D J. The "New" Science of Networks[J]. Physics Today, 2003, 6(5):243 270

15. Axelrod R, Hamilton W D. Evolution of Cooperation[M]// The evolution of inequality :. Stanford University Press, 1981:135-60.

16. Nowak M A, May R M. Evolutionary Games and Spatial Chaos.[J]. Nature, 1992, 359(6398):826 829

17. Hamlin A, Sugden R. The economics of rights, co-operation and welfare[J]. 1986, 97 